\title{
The progress of AAV-mediated gene therapy in neuromuscular disorders
}

\author{
Abstract \\ Introduction: The well-defined genetic causes and monogenetic nature of many neuromuscular \\ disorders, including Duchenne muscular dystrophy (DMD) and spinal muscular atrophy (SMA), \\ present gene therapy as a prominent therapeutic approach. The novel variants of adeno-associated \\ virus (AAV) can achieve satisfactory transduction efficiency of exogenous genes through the \\ central nervous system and body-wide in skeletal muscle.
}

Areas covered: In this review, we summarize the strategies of AAV gene therapy that are currently under preclinical and clinical evaluation for the treatment of degenerative neuromuscular disorders, with a focus on diseases such as DMD and SMA. In addition to gene replacement strategy, we provide an overview of other approaches such as AAV-mediated RNA therapy and gene editing in the treatment of muscular dystrophies.

Expert opinion: AAV gene therapy has achieved striking therapeutic efficacy in clinical trials in infants with SMA. Promising results have also come from the preclinical studies in small and large animal models of DMD and several clinical trials are now on the way. This strategy shows great potential as a therapy for various neuromuscular disorders. Further studies are still required to confirm its long-term safety and improve the efficacy.

\section{Keywords}

Adeno-associated virus, gene therapy, gene editing, muscular dystrophy, neuromuscular disorders, RNA therapy, spinal muscular atrophy. 


\section{Article Highlights}

- Recombinant adeno-associated virus (rAAV) vectors are efficient in transducing exogenous genes body-wide following systemic delivery, with target organs including skeletal muscles, cardiac muscles and motor neurons.

- AAV gene therapy represents a promising approach for treating Duchenne/Becker muscular dystrophy (DMD/BMD), spinal muscular atrophy (SMA) and Myotubular Myopathy.

- The successful clinical trial of scAAV9-SMN (AVXS-101) shows striking efficacy and it is among the most promising therapeutic approaches for the treatment of infants with type I SMA.

- In addition to gene replacement, other approaches such as AAV-mediated RNA therapy and gene editing have also shown potential in the treatment of neuromuscular diseases. 


\section{Introduction}

Gene therapy is based on the introduction of exogenous nucleic acids within living cells to achieve a therapeutic effect to treat or intervene particular disorders. The genomic material can be delivered by viral and non-viral vectors. The selection of the delivery system varies depending on a number of factors, such as the type of tissues to be targeted, the size of the genomic material to be delivered and the duration of the transgene expression in order to reach a therapeutic effect.

Neuromuscular disorders are a group of inherited and acquired conditions that primarily affect one or more components of the neuromuscular unit, including motor neurons and skeletal muscle. For many of these conditions, including Duchenne muscular dystrophy (DMD) and spinal muscular atrophy (SMA), the genetic causes are well defined. Gene therapy therefore holds promise for the treatment of these monogenic neuromuscular disorders for which the development of novel treatments is urgent and challenging. While numerous viral vehicles have been investigated for gene therapy, to date the most promising vectors for the treatment of neuromuscular disorders derive from adeno-associated viruses (AAVs) [1]

\section{Adeno-associated virus AAV}

AAV was first discovered as a contaminant of adenovirus preparation in 1965. The virus is a defective parvovirus, its infection or replication requires helper genes (E1A, E1B, E4 and E2A) provided by herpes simplex virus or adenovirus [2]. In the absence of a helper virus, AAV establishes latency by integrating into AAVS1 locus on human chromosome 19 (19q13) [3]. AAVs are small icosahedral viruses with a non-enveloped capsid and linear single-strand DNA (ssDNA) genome in which three open reading frames (ORFs), rep (Replication), cap (Capsid) and aap (gene encoding the assembly activating protein) are flanked by two inverted terminal repeats (ITRs). ITRs act as origins of genome replication and they are required for integration and genome packaging [4]. The rep gene encodes four non-structural proteins (Rep78, Rep68, Rep52 and Rep40) involved in 
viral replication and integration, while the cap gene encodes three viral capsid proteins (VP1, VP2 and VP3) from ORF1 and AAP protein from ORF2. AAP protein is essential for AAV capsid assembly by stimulating VP proteins transport to the nucleolus and by playing a role during the assembly process [5].

The introduction of exogenous DNA into human and mouse tissue culture cells with a recombinant, AAV-based vectors (rAAV) was first described in 1984 [6]. For the production of single strand AAV (ssAAV) vectors, rep and cap genes are replaced with the foreign DNA inserted between ITR sequences. ITRs are kept since they are the only elements required in cis to ensure packaging of the vector genome, while rep and cap genes and the minimal elements from a helper virus are provided in trans [7]. rAAV represent one of the most promising viral vector systems for gene therapy. In December 2017, Luxturna (Spark Therapeutics Inc), became the first AAV gene therapy approved by the U.S. Food and Drug Administration (FDA) for the treatment of patients with Leber's congenital blindness caused by biallelic RPE65 mutation (https://www.fda.gov/NewsEvents/Newsroom/PressAnnouncements/ucm589467.htm). Further success in AAV gene therapy is demonstrated by the promising outcomes of clinical trials for Becker muscular dystrophy, heart disease, Parkinson's disease, cystic fibrosis and haemophilia [911]. The wide use of these vectors is based on their safety profile, ability to remain in host cells as episomal DNA with negligible integrating rate and the capability to infect both dividing and nondividing cells. In addition, it is possible to primarily target specific subsets of cell types in vivo due to the specific tropism of the different AAV-serotypes. To date, there are 13 naturally occurring AAV human serotypes and more than 100 nonhuman primates serotypes, which have shown tissue transduction preferences [12].

Within ssAAV vectors, a single strand DNA of maximum 4.7 kilobases $(\mathrm{kb})$ is packaged. Once inside the cell, the cellular DNA polymerase synthesizes the second strand and multiple vector genomes then forms concatemers of circular DNA [13]. In ssAAV, the synthesis of the 
complementary strand significantly reduces the speed of transgene expression. Self-complementary adeno-associated virus (scAAV) vectors, where both DNA strands are packaged, allow faster transgene expression and increase in transduction [14] but at the expenses of a further reduced packaging capacity of $\sim 2.2 \mathrm{~kb}$ (Figure 1 ). Notably, the limited packaging capacity is one of the most significant limits of scAAV vectors which make this strategy not suitable for large gene delivery. Another limit of AAV gene therapy is the gradual dilution of the delivered transgene in dividing cells which implicates that a re-administration may be required later in life if the target tissues are in active regeneration or proliferation. This issue is less crucial in non-dividing cells in which the transgene expression is more stable after a single injection. Recently, to overcome this limit and achieve long-term transgene expression in proliferating cells, the addition of a scaffold/matrix attachment region (S/MAR) to AAV vectors has been attempted and showed promising in vitro results that will have to be confirmed in vivo in animal models [15].

\section{Gene therapy with AAV vectors in neuromuscular disorders}

\subsection{AAV-gene therapy in Duchenne Muscular Dystrophy}

DMD is one of the most common human genetic disorders, affecting approximately 1:5000 live births, characterized by progressive muscle wasting [16]. DMD is an X-linked recessive disorder due to mutations (deletions, insertions, duplications and point mutations) in the dystrophin gene, leading to the disruption of the open reading frame followed by the production of a non-functional protein. The dystrophin gene consists of 79 exons and encodes for a component of the highly conserved dystrophin-associated glycoprotein complex (DGC), essential for the stability and function of myofibres in skeletal and cardiac muscle $[17,18]$.

A number of gene therapy approaches have been investigated to date, and one of the most promising approaches to treat DMD is the introduction of a truncated but functional dystrophin protein using AAV vectors which can efficiently infect both skeletal and cardiac muscles (Figure 
2).

\subsubsection{Gene replacement}

Due to the very large size of the dystrophin gene, $2.2 \mathrm{MB}$ genomic sequence and $14 \mathrm{~kb}$ coding sequence, it is impossible to package the entire dystrophin cDNA in currently available AAV vectors. The rationale behind the approach of introducing a truncated but functional dystrophin product for DMD derives from the observation that Becker patients with short but in frame dystrophin genes can present a mild dystrophic phenotype [19]. This is believed to be due to the presence of dispensable domains in the central region of the dystrophin protein. By excluding several non-essential domains, smaller but still functional human dystrophin genes have since been created [20]. Micro-dystrophin with a size smaller than $5 \mathrm{~kb}$ can then be packaged into AAV vectors.

Local and systemic injections of micro-dystrophin AAV vectors in the dystrophin-deficiency $m d x$ mice restored functional dystrophin and dystrophin-glycoprotein complex, as well as muscle histopathology and function [21-23]. Systemic delivery of micro-dystrophin AAV vectors in the dog models of DMD has also demonstrated the safe and efficient transduction of the functional dystrophin gene [24]. The promising results obtained with AAV-micro-dystrophin gene treatment in animal models have led to their clinical applications in DMD patients. However, the first clinical trial of AAV micro-dystrophin gene (rAAV2.5-CMV-minidystrophin, d3990) through intramuscular delivery (NCT00428935, 2007-2013), did not show the same promising results as previously observed in animal models. The study was performed in six patients with frame-shift deletions in $D M D$ gene. Each patient received an injection of rAAV2.5-CMV-minidystrophin in one arm and placebo in the other arm. Ninety days after the treatment, no micro-dystrophin expression was detected in muscle biopsy specimens. The exogenous protein was detected only in two patients that were examined forty-two days after the injection. Moreover, in two boys, due to 
the presence of pre-existing antibodies to dystrophin protein, an immune response against dystrophin was detected, and an immune response against the AAV vector was also observed in one patient. Contrary to what was observed in mouse models, this clinical trial showed a strong immune response against transgene and/or AAV proteins capsid. Although this response was at least partially triggered by using a CMV promoter and a chimeric engineered capsid, this study pointed out the necessity of pre-screening patients for related antibodies, before admission into trials, since the failure to maintain long-term transgene expression is secondary to the immune response $[25,26]$. A recently completed phase I clinical trial (NCT02376816, 2015-2017), took advantage of these information and was planned to intramuscularly inject a different micro-dystrophin driven under the control of a muscle-specific creatine kinase (MCK) promoter in a new AAV serotype (rAAVrh74.MCK.microdystrophin), with the aim of eluding or attenuating the immune response observed in the first clinical trial. The results are yet to be published.

The strong immunological reactions observed in the first DMD clinical trial, were also found in canine models of DMD (GRMD) following the AAV2, AAV6, AAV8 and AAV9 vectors administration, resisting the transgene expression [27-30]. The undesired immune response can be bypassed by the use of transient immunosuppression drugs [31]. Interestingly, this occurs only in adult dogs and no toxicity or immune response events have been described in neonatal dogs. However, although no toxicity or immune response was observed in neonatal dogs, muscle atrophy, delays in growth and loss of cardiac tropism have been detected in these dogs [27,28]. Recently, promising results have been achieved with the use of rAAV2/8-Spc12-cMD1 vector in 12 juvenile GRMD dogs, in which an optimized microdystrophin transgene under the control of a muscle- and cardiac- specific promoter was used [29,30]. The safety and efficacy of both intramuscular and intravascular administrations of this vector were demonstrated. Long-term transgene expression with amelioration of histopathology and muscle functional improvement were presented without any immune response [30]. Similarly, in another study, the efficiency and safety of intamuscular 
and intravenous injection of AAV8.MCK.micro-dystrophin.FLAG vector have been shown in nonhuman primates. Transgene expression was detected five months post-injection, with elevated expression of the truncated dystrophin protein in almost all muscle fibers [32]. Several clinical trials using different AAV serotypes, micro-dystrophin genes and promoters are now underway (table 1).

In addition to the delivery of the dystrophin gene itself, surrogate AAV-gene therapy approaches for the treatment of DMD have also been investigated with positive results reported in dystrophic animal models. These include the over-expression of the cytotoxic $\mathrm{T}$ cell GalNAc Transferase (GALGT2 gene) by targeting the dystroglycan complex [33], over-expression of follistatin to increase muscle mass and prevent muscle degeneration [34], and expression of micro-utrophin, a shorter version of the dystrophin paralog $[35,36]$.

The therapeutic effects of GALGT2 AAV-gene therapy have been demonstrated in small and large animal models. The delivery of GALGT2 gene leads to an overexpression of GALGT2 enzyme, a protein responsible for glycosylation of $\alpha$-dystroglycan in skeletal muscle. Moreover, GALGT2 overexpression leads to an increment of dystroglycan binding proteins, protecting dystrophic muscle from injury and inhibiting the development of muscular dystrophy [37]. A phase I/II clinical trial (NCT03333590) of rAAVrh74.MCK.GALGT2 gene therapy began in 2017 and is currently recruiting patients. It is an open-label, dose escalation trial with the aim of assessing the safety of intravascular limb infusion technique in both legs of DMD patients.

Follistatin is a muscle growth stimulating protein that acts by inhibiting the myostatin pathway and inducing muscle hypertrophy [38]. Overexpression of follistatin by AAV-gene therapy has displayed remarkable results in both pre-clinical studies and clinical trial in BMD (where a shorter form of dystrophin is partially expressed) and patients with Sporadic Inclusion Body Myositis (SIBD) (NCT01519349) [11,39,40]. In BMD patients, administration of rAAV1.CMV.huFollistatin344 vector resulted in a significant improvement in histopathological and functional outcomes with an increase in muscle strength and gain in the 6 Minute Walk Test 
(6MWT), in the absence of any immune response [11]. Follistatin AAV-gene therapy was tested in another phase II clinical trial in six DMD patients by multiple intramuscular injections (NCT02354781). Results are not available yet. However, it is still under debate if this treatment could be beneficial when applied to DMD patients, as larger muscle fibres depleted of dystrophin may actually be weaker and more fragile than smaller myofibres [41]. A recent report on the existing endogenous down-regulated myostatin signalling pathway in patients with DMD and other muscle-wasting conditions may provide an explanation for the low clinical efficacy of antimyostatin approaches in several of the clinical trials and the apparent contradictory results between mice and patients [42].

Although promising, surrogates AAV-gene therapy strategies do not correct the genetic cause of the disease, but they could be used as adjuvant therapies in combination with dystrophin-restoration approaches [43].

\subsubsection{AAV-mediated CRISPR/Cas9 therapy}

Genome editing is potentially an appealing therapeutic approach for DMD: here the mutant dystrophin is not replaced but is modified by CRISPR/Cas9 technology delivered by AAV vectors. Studies from several research groups have provided the proof-of-concept that AAV vector is an efficient method for genome editing delivery.

The possibility to restore truncated dystrophin expression with genome editing in both skeletal and cardiac muscle in $m d x$ mouse has been demonstrated [44-47]. In this system, exon 23 which contains the nonsense mutation is effectively excised, leading to the restoration of ORF and production of a truncated but functional protein. In order to achieve an effective CRISPR complexes delivery, a single and a dual-AAV vectors system were designed. The dual system consists of an AAV vector expressing SaCas9 nuclease and a second AAV vector containing two single-guide RNA (gRNA) expression cassettes. The therapeutic effects were detected in both adult 
and neonatal mice, following local or systemic deliveries. Dystrophin restoration was maintained for at least six months after treatment in $m d x$ mice. A modest efficiency in myogenic stem cells transduction has also been reported, which may represent an alternative cell therapy strategy for the treatment of DMD [45,48]. In addition to the dual system, a single approach was generated to evaluate the cardiac function after the restoration of dystrophin expression. SaCas9/gRNA constructs were packaged into AAV serotype rh74 and delivered in neonatal and adult $m d x / U t r^{+/-}$ mice. After a single systemic delivery, dystrophin protein was restored in $40 \%$ of cardiac muscle with reduction in fibrosis and a significant functional improvement [47].

Another approach has been tested in the $m d x^{4 c v}$ mouse model, which harbours a nonsense mutation in exon 53. In this case, deletion of exon 52 and 53 restores the ORF. Excision of these two exons has been achieved by generating either a single (expressing both SaCas9 and two sgRNA) or a dual (two vectors, expressing either SpCas9 or two sgRNA) AAV6 vectors systems. In both cases, Cas9 expression was restricted to skeletal and cardiac muscles under a CK8 promoter to reduce possible off-target events in non-muscle cells. Widespread dystrophin expression, observed after both local and systemic deliveries, led to an improvement of dystrophic histopathology [49].

Results obtained from these studies are encouraging and CRISPR/Cas9 gene editing delivered by AAV vectors could potentially be developed as a treatment for DMD. Moreover, this technology can be used for a wide range of mutations and it was estimated that almost $60 \%$ of DMD patients could be treated [50]. However, more studies are needed to prove the safety of this approach as the undesired off-target effects may hurdle its clinical translation.

\subsubsection{AAV-mediated antisense oligonucleotide therapy}

AONs are widely used to modulate the pre-mRNA splicing in dystrophin gene, to restore the disrupted reading frame by exon-skipping strategy. Two exon-skipping AONs (Eteplirsen and Drisapersen) have been extensively investigated in clinical trials (NCT02255552, NCT01540409, 
NCT02420379, NCT01396239, NCT03218995, NCT02286947, NCT02636686 and NCT01803412). Intramuscular administrations of these AONs were generally well tolerated, and led to improvement in the 6MWT with the restoration of truncated dystrophin at a low level followed by restoration of DGC complex [51-53]. Although these AONs have presented promising results in preclinical and clinical studies, improvement in this technology is still required in order to increase exon-skipping efficacy in skeletal and cardiac muscles.

To improve the delivery of AONs and exon-skipping efficiency in skeletal and cardiac muscles, modified small nuclear RNAs (snRNAs) constructs have been generated to carry AONs to enter the nucleus, by AAV vectors delivery. U7 small nuclear RNA (U7 snRNA) is one of the snRNAs used for this new AONs approach. U7 snRNA is involved in histone pre-mRNA 3'-end processing. The modification consists of the replacement of U7 Sm binding site (U7 Sm WT) with the consensus sequence derived from snRNPs (U7 SmOPT), leading to a more efficient accumulation in the nucleus [54]. Moreover, the complementary sequence to the histone downstream element (HDE) is replaced with the specific antisense sequence. Thus, once inside the nucleus the original function of U7 snRNA is lost, but will be able to carry a specific antisense sequence. The advantage of using U7 SmOPT is that AONs are transported directly to where the target pre-mRNAs are located, protecting from degradation.

The engineered U7 snRNA carrying specific exon-skipping AONs delivered by AAV vectors have been used in $m d x$, utrophin/ dystrophin double knockout (dKO) mouse and canine models with promising results. After a single intravenous administration of AAV-U7-SD23/BP22 vector in $m d x$ and dKO mouse models, dystrophin expression was restored in all muscles analysed, including in the heart with improved cardiac function. The rescue of DGC complex, the attenuation of muscle regeneration and necrosis, and an extension of the lifespan from 10.2 to 50.2 weeks, have been observed $[55,56]$. The use of the same strategy in GRMD canine model with two different AAV vectors (AAV1 and rAAV6) also showed notable results. The dystrophic features have been 
reduced with improvement in muscle strength without any immune response against the rescued dystrophin and/or AAV proteins capsid during the five-year follow up. However, the number of corrected muscle fibers decreased with time suggesting the requirement of recurrent treatments which may limit the clinical translation of this approach $[57,58]$.

\subsection{AAV-gene therapy in Spinal Muscular Atrophy}

SMA is one of the most common genetic diseases in childhood and the leading cause of infant mortality. It is characterized by muscle weakness and paralysis secondary to spinal motor neuron death. SMA is an autosomal recessive disease caused by mutations in the Survival of Motor Neuron

gene $1(S M N 1)$, localized in chromosome 5. SMN genes consist of two paralogue genes: SMN1 and SMN2, the telomeric and the centromeric copies, respectively. SMA, in 95\% of cases, is due to the loss-of-function mutations in SMN1 gene, while SMN2 remains intact in all patients. Even though both genes encode for SMN protein, the presence of a single nucleotide transition $(C>T)$ in exon 7 of SMN2 gene leads to exon 7 skipping in $90 \%$ of transcripts and unstable protein products. SMN2 gene cannot fully compensate the role of SMN1 gene as only $10 \%$ of SMN2 transcripts can translate into a functional SMN protein [59].

For a gene therapy treatment of SMA, the most suitable viral vector is AAV and more precisely AAV9 serotype. AAV9 is able to cross the blood-brain barrier (BBB), allowing intravenous administration rather than intrathecal injection into the spinal cord, as is currently used in Spinraza AON drug treatment $[60,61]$. So far, two AAV-gene therapy approaches have been validated for the treatment of SMA: the SMN1-AAV gene replacement and the AAV-mediated AON approaches (Figure 3).

\subsubsection{Gene replacement}

The monogenetic nature of SMA and the relatively short coding sequence of SMN1 gene have made SMA the perfect disease target for AAV-mediated gene therapy. Preclinical studies using scAAV9 
vectors result in significant improvement in lifespan and phenotype rescue in SMA transgenic mice. The complete rescue of the disease phenotype in SMA transgenic mice after a single scAAV9SMN1 administration has been reported by several groups, with mice survived for over 200 days [61-64]. While most of the studies were conducted in neonatal mice when the BBB is still penetrable, one study delivered a single intramuscular injection of scAAV9 vectors in adult mouse and showed widespread viral vector transduction, including the whole spinal cord and peripheral organs [63]. This result indicates the great potential of scAAV9 in the development of gene therapy strategies for neurological diseases where the primary target is motor neurons. Promising results have also been achieved in larger animals, including pig and non-human primates (NHPs) following scAAV9-SMN administration. Widespread motor neuron transduction was observed in NHPs and efficient SMN expression was achieved in motor neurons of treated pig and monkeys $[65,66]$.

The first scAAV9-SMN clinical trial (NCT02122952) was started by AveXis Inc in 2014 (Table 1). This open-label, dose-escalation phase I clinical trial was designed to evaluate safety and efficacy of intravenous delivery of AVXS-101 as a treatment for patients with type I SMA. Fifteen patients with type I SMA received a single intravenous dose of scAAV9-SMN. Patients were divided in two cohorts: low-dose cohort (three patients at $6.7 \times 10^{13} \mathrm{vg}$ per kilogram of body weight) and high-dose cohort (twelve patients at $2.0 \times 10^{14} \mathrm{vg}$ per kilogram). All 15 patients $(100 \%)$ were alive and eventfree at 20 months of age, as compared with a rate of survival of $8 \%$ in an historical cohort. AVXS101 was well tolerated in all patients. Transient and asymptomatic elevation in serum aminotransferase levels were found in four patients and were resolved with prednisolone treatment. In the high dose cohort, striking motor function improvement was achieved in all treated patients, including capabilities of unassisted sitting, oral feeding, speaking and even independent walking $[67,68]$. There was no report of clinical regression in motor function in a two-year follow-up in this study, although further studies are still required to assess the long-term safety and durability of this gene-replacement in SMA patients. In September 2017, an open-label phase III clinical trial of 
AVXS-101, known as STRIVE (NCT03306277), was started. In December 2017, AveXis announced another phase I clinical trial based on intrathecal administration of AVXS-101 in patients with SMA type II or III (NCT03381729) (Table 1). This trial aims to evaluate the safety and tolerability of the administration into the central nervous system (CNS) of AVXS-101 in older patients with less severe clinical features. Patients will be stratified in two groups, those $<24$ months and between 24-60 months of age at the time of dosing. Different from the previous trials, the enrolled patients are able to sit but cannot stand or work at the time of study entry. The intrathecal administration is expected to deliver SMN1-AAV efficiently in the CNS and overcome the issue of the high amount of virus required when administrated intravenously.

Very recently, severe toxicities in high dose AAV gene therapy when using neurotropic AAV9 variants to target CNS via systemic delivery in large animals were reported $[69,70]$. Intravenous injection of AAVhu68 vector carrying a human SMN1 transgene at $2 \times 10^{14}$ genome copies /kilogram of body weight, a dose similar to that employed in the SMA clinical trials described above, was delivered to three juvenile NHPs (aged 14 months) and three piglets (aged 7-30 days). Severe toxicities, including elevated transaminase, acute liver failure, degeneration of dorsal root ganglia sensory neurons, occurred in both NHPs and piglets [69]. This study raises concern about the safety profile of AAV vectors when high dose of viral vectors are required to target CNS and skeletal muscles by intravenous injection. In support of this finding, the same group also reported additional data on another AAV9-like vector, PHP.B, in a NHP (N=1). Although different AAV9like capsid and a different transgene were utilized, the toxicology profile was similar when AAVPHP.B vectors were delivered intravenously at high dose $\left(7.5 \times 10^{13}\right.$ genome copies /kilogram $)$ [70]. These studies highlighted the need of additional work to understand the mechanism of the systemic and sensory neuron toxicity observed in the large animal studies, and to assess the relevance of such toxicity in human trials. In addition, it is important to point out that capsid and transgene in these studies were similar but not identical to those used in SMA human trials [67]. 
These findings need to be handled by the gene therapy community in a balanced and responsible fashion, and need to be supported by more studies from different independent groups [71].

\subsubsection{AAV-mediated antisense oligonucleotide therapy}

In addition to gene replacement approach with the introduction of exogenous SMN1 to achieve the production of functional SMN protein, it is possible to increase full-length $S M N$ transcript expression acting through SMN2 gene. SMN2 gene is a target for the treatment of SMA disease since all patients carry at least one copy. This can be achieved by delivering specific AONs targeting splicing motifs [72,73]. Spinraza, an AON drug to correct the splicing of SMN2 gene and increase SMN protein expression, has become the first drug approved by the FDA for the treatment of SMA and is now in market [74]. However, to achieve the desired therapeutic effect, the AON drug needs to be administrated repeatedly by intrathecal injections for life.

To maintain a long-term splicing correction, AON engineered with U1 and U7 snRNA has been conjugated with scAAV9 vector and tested in SMA mouse models. The modified U1 snRNA (ExSpeU1) was generated by the substitution of its 5'-end with an AON sequence targeting the intronic splicing silencer N1 (ISS-N1) [75]. A single intraperitoneal injection of scAAV9-ExSpeU1 sm21 in newborn SMA transgenic mice showed a notable exon 7 inclusion in SMN transcript in all the tissues analysed (skeletal muscle, liver, kidney, heart and brain), suggesting that this approach could be a promising therapy for SMA [76].

In another study, a construct with modified U7 snRNA gene carrying single stranded AON complementary to $S M N$ exon 7 and an additional sequence able to recruit the positive splicing factor SRSF1 (U7-ESE-B) has been generated to stimulate exon 7 inclusion and SMN protein production [77,78]. The construct of scAAV9 vectors containing four tandem copies of the U7ESE-B gene significantly increased the lifespan and muscle functions when injected into the cerebral ventricles of newborn SMA mice, with results comparable to the treatment of SMN1 
cDNA-scAAV9 gene therapy and splicing-switching-AON therapy. However, it should be noted that a slightly higher dose of the scAAV9-4xsU7 vectors $\left(4 \times 10^{13} \mathrm{vg} / \mathrm{kg}\right)$ compared to the scAAV9SMN1 cDNA vectors $\left(2 \times 10^{13} \mathrm{vg} / \mathrm{kg}\right)$ was needed to increase the average survival of the SMA transgenic mice beyond 100 days [78,79]

\subsection{AAV-gene therapy in other Muscular Dystrophies}

\subsubsection{AAV-gene therapy in Oculopharyngeal Muscular Dystrophy (OPMD)}

OPMD is a late-onset autosomal dominant muscle disease. It is characterized by specific and symmetrical muscles involvement with a slowly progressive course. The primary symptoms include ptosis of eyelids, secondary to weakness of the levator palpebrae, and dysphagia, due to the involvement of pharyngeal musculature and proximal limb weakness [80]. The causative gene, called polyadenylate binding protein nuclear 1 (PABPN1) gene, encodes for a ubiquitous polyadenylation factor involved in RNA post-transcriptional processing [81]. OPMD patients harbour 11-18 trinucleotide (GCN) expansion, instead of the normal 10 in PABPN1 exon 1 leading to the formation of 12-17 alanines in the mutant protein. The mutated protein, is unable to fold correctly and hence generates insoluble filamentous intranuclear inclusions (INIs) within the muscles [82]. No cure is currently available to this condition. Very recently, a dual-AAV gene therapy approach was reported to rescue many pathological features of the disease [83].

This dual approach consists of knocking down the endogenous PABPN1 by RNA interference (shRNA) and replacing the protein with a normal, wild-type PABPN1. To achieve a rapid and efficient PABPN1 knockdown, three shRNAs into a tricistronic expression cassette (shRNA3X), with each hairpin RNA driven by a different polymerase III promoter, were cloned into the selfcomplementary AAV8 backbone (scAAV-shRNA3X). The expression cassette for codon-optimized wild-type PABPN1 sequence (optPABPN1), which is resistant to shRNA3X knockdown, was packaged as a single-stranded construct into AAV9 (ssAAV-optPABPN1). A single intramuscular 
injection was performed in A17 OPMD mice at ten to twelve weeks of age. Outcomes on skeletal muscle pathology and function were analysed at 18 weeks post-injection. Significant phenotype rescue was obtained with the co-expression of scAAV8-shRNA3X and AAV9-optPABPN1, which reduced the amount of insoluble aggregates, decreased muscle fibrosis, reverted muscle strength to the level of healthy muscles and normalized the muscle transcriptomes. This approach has been recently optimized by Benitec Biopharma using a single vector system (BB-301) including both shRNAs and optPABPN1 under the control of a muscle-specific promoter. The single vector shows efficacy as great as the dual-vectors approach, with restoration of muscle force, muscle weight, clearance of INIs and reduction of fibrosis. At the beginning of 2017, the European Commission has granted Orphan Drug Designation to BB-301 as potential treatment of OPMD patients. The first clinical trial is due to start in the second half of 2018 (Figure 4) [84].

The dual-AAV gene therapy strategy developed for OPMD suggests that a similar approach could be used in other dominant gain-of-function neuromuscular conditions where silencing the endogenous mutant gene and introducing the wild-type copy are required to correct the disease.

\subsubsection{AAV-gene therapy in X-Linked Myotubular Myopathy (XLMTM)}

X-linked myotubular myopathy (XLMTM) is a rare neuromuscular disorder belonging to the group of congenital myopathies (CMs). XLMTM represents a fatal condition in which the first symptoms usually appear at birth with muscle weakness, hypotonia, peculiar facial features, feeding difficulties and respiratory insufficiency [85]. XLMTM disorder is caused by mutations in myotubularin (MTM) gene, which is mapped to chromosome Xq28, resulting in loss of function of the encoded myotubularin protein [86]. Myotubularin is a phosphoinositide lipid phosphatase functions in muscle fibers maintenance [87,88]. Dysfunction or lack of this protein leads to disorganization of skeletal muscle fibers, characterized by rounded shape and the presence of central nuclei surrounded by organelles suggesting an arrest in differentiation process [89]. 
No specific treatment is available to date for patients with myotubular myopathy. AAV-gene therapy to introduce exogenous myotubularin has been attempted in a XLMTM mouse model by a single intramuscular injection of rAAV2/1-CMV-Mtm. Myotubularin replacement in mice largely corrected nuclei and mitochondria position in myofibers and led to a strong increase in muscle mass and recovery of muscle force [90]. Similarly, systemic administration of a single dose of rAAV8MTM1 vectors in Mtm1-deficient mice and in XLMTM dogs resulted in robust improvement in motor activity and muscle function, corrected muscle pathology and prolonged survival in the absence of toxicity or immune response, although a decrease of transgene expression was observed at four years after treatment [91,92]. These promising pre-clinical studies demonstrate the therapeutic potential of AAV-mediated gene therapy for MTM in small- and large- animal models, and provide proof-of-concept for the clinical translation to XLMTM patients.

A phase I/II clinical trial, called ASPIRO (NCT03199469), is currently conducted by Audentes Therapeutics (Table 1). Children aged less than 5 years with XLMTM have been treated with a single intravenous dose of AT132 vector (rAAV8-MTM1) and will be followed for safety and efficacy for 5 years. In January 2018, Audentes has announced positive interim data for the first 12 weeks of the first cohort of children treated with $1 \times 10^{14}$ vg per kilogram of AT132 vector. In addition to a good safety profile, significant improvements in neuromuscular function, respiratory function and multiple developmental milestones are all achieved at 12 weeks in the first treated patient. While the trial is still at its early phase, these promising data indicate the potential of AAVgene therapy in the treatment of XLMTM (from Audentes Therapeutics's media release).

\section{Expert Opinion}

AAV vectors are currently the most widely used viral vectors in gene therapy for neuromuscular disorders, due to their good safety profile, lack of genome integration and efficient gene transduction in neuromuscular components. The skeletal muscle tissue can be efficiently transduced 
by several AAV serotypes such as AAV6, AAV8 and AAV9 [1]. Furthermore, neuromuscular disorders can benefit from the long-term transgene expression of AAV in non-dividing cells, such as skeletal muscle fibres and motor neurons $[22,93]$. AAV has hence become the most preferred vector in advanced preclinical gene therapy studies and early phase clinical trials in neuromuscular diseases, such as SMA, XLMTM, DMD and OPMD.

The scAAV9-SMN1 clinical trial in SMA is so far the most successful gene therapy trial conducted in neuromuscular conditions. Facilitated by the promising primary outcomes achieved from the previous phase 1 clinical trial, it has then moved rapidly into phase 3 [67]. Systemic administration of scAAV9-SMN is advantageous for not only crossing the BBB and leading to efficient biodistribution in central nervous system, but also targeting peripheral organs and providing SMN protein body-wide. Increasing evidences suggest that peripheral organs (e.g., autonomic and enteric nervous systems, cardiovascular system, and liver etc. [94-96]) are also involved in the pathogenesis of SMA, in addition to the primary defect in spinal motor neurons. Indeed, restoration of SMN protein using AON therapy by targeting both systemic organs and central nervous system in SMA transgenic mice shows greater therapeutic efficacy than the CNS exclusive restoration [97]. However, while scAAV9-SMN gene therapy is supposed to be a one-off treatment for SMA, it is still unknown how long the expression of the transgene may persist in CNS and peripheral organs, and if a repeat treatment is required later in life and what strategy will be used. Long-term studies on safety become crucial in light of the recent reports on safety concerns raised in systemic delivery of high-dose neurotrophic AAV9 vectors in large animals $[69,70]$.

Preliminary results from the current ASPIRO clinical trial for XLMTM, though related to a very short time study (12 weeks) in a small number of patients, are impressive. It is worthy to note that for XLMTM a small amount of transgene expression is sufficient to make a substantial difference and induce a positive clinical outcome [91]. In contrast to SMA and XLMTM, treating DMD is likely to be more challenging with the current gene replacement and AAV-mediated AON 
approaches as dystrophin has a structural role: a functional protein has to be generated at high level to sustain the myofibres from collapsing. Current research is mainly focused on improving the functionality of the micro-dystrophin conformations (e.g. including the domains with crucial roles) and its expression (e.g. using more efficient regulatory sequences and by codon optimization in human). Alternatively, other strategies have been tested in preclinical applications to transfer the full-length dystrophin gene using dual high-capacity AAV vectors [98], or splitting the full coding sequences into three fragments and packaging them into separate independent vectors which are able to reconstitute a larger molecule in vivo $[99,100]$. In DMD studies, the transduced microdystrophin gene product, if functional and highly expressed, is able to stabilise the muscle turn-over and be maintained in muscle fibres for many years, as shown in murine preclinical models [22]. Information on the pathological and clinical outcomes of expressing high levels of micro-dystrophin genes in human muscle from currently ongoing clinical trials may illuminate this.

The dual-AAV-gene therapy developed for OPMD can reduce significantly the cost of the vector and facilitate the regulatory pathway. Embedding the shRNA molecules into a pre-existing microRNA structure and the possibility to drive the shRNA expression with a muscle specific promoter are expected to make the vector safer. If this approach is approved to be safe and efficacious in clinical trial, it would become very meaningful for other dominant gain-of-function genetic conditions that could benefit from the same strategy [83].

Although promising in preclinical studies, the development of AAV-mediated AON therapies is currently behind the gene replacement approach as the molecular pathways from cells transduction to the protein expression is complex, and may require higher amounts of AAV vectors. Both gene replacement and AAV-mediated AON therapy will likely require the re-administration of the vector at later stage. This issue currently is unresolved and becomes urgent to address.

Furthermore, more work is needed to further investigate the potential risks of high systemic dosing 
of AAV when treating neuromuscular disorders, as recently suggested. The dose of the vector administered requires rigorous preclinical safety studies in large animals to assess both the clinical outcome and the possible systemic toxicity [69, 70]. Meanwhile, a more comprehensive understanding of the underlying mechanisms of the toxicities reported so far and collaborative investigations on this issue from different independent groups will undoubtedly benefit the entire AAV gene therapy community.

The future of gene therapy also lies in novel technologies, such as gene editing that would not require vector re-administration. Genome editing using CRISPR/Cas9 system has demonstrated remarkable potential for neuromuscular disorders in preclinical studies. However, the low gene modification rates in skeletal muscle in vivo may still restrict its clinical translation at the current stage [44]. Further studies and improvement on this young technology are required to facilitate its bench to bedside translation.

In conclusion, while the recent ground-breaking clinical success of Luxturna, the first FDA approved gene therapy drug for inherited blindness, has been deemed a medical milestone, the promising outcomes from early phase clinical trials in SMA and XLMTM further reinforce the evidence that AAV gene therapy holds great potential for the treatment of neuromuscular conditions. We are now entering a new AAV-gene therapy era.

\section{Acknowledgments}

This work was supported by the NIHR Great Ormond Street Hospital Biomedical Research Centre (GOSH BRC). The views expressed are those of the author(s) and not necessarily those of the NHS, the NIHR or the Department of Health. The authors declare no conflicts of interest. 


\section{Bibliography}

Papers of special note have been highlighted as either of interest $(\bullet)$ or of considerable interest $(\bullet)$ to readers.

[1] Wang D, Zhong L, Nahid MA, Gao G. The potential of adeno-associated viral vectors for gene delivery to muscle tissue. Expert Opin Drug Deliv 2014;11:345-64

[2] Atchison RW, Casto BC, Hammon WM. Adenovirus-associated defective virus particles. Science (80-. ) 1965;149:754-6

[3] Kotin RM, Linden RM, Berns KI. Characterization of a preferred site on human chromosome $19 \mathrm{q}$ for integration of adeno-associated virus DNA by non-homologous recombination. EMBO J 1992;11:5071-8

[4] Koczot FJ, Carter BJ, Garon CF, Rose JA. Self-complementarity of terminal sequences within plus or minus strands of adenovirus-associated virus DNA. PNAS 1973;70:215-9

[5] Sonntag F, Schmidt K, Kleinschmidt JA. A viral assembly factor promotes AAV2 capsid formation in the nucleolus. PNAS 2010;107:10220-5

[6] Hermonat PL, Muzyczka N. Use of adeno-associated virus as a mammalian DNA cloning vector: transduction of neomycin resistance into mammalian tissue culture cells. PNAS 1984;81:6466-70 - This article provides for the first time the evidence that AAVs can be used as delivery system for the introduction of foreign DNA into human and mouse tissue cultured cells.

[7] Samulski RJ, Chang LS, Shenk T. Helper-free stocks of recombinant adeno-associated viruses: normal integration does not require viral gene expression. J Virol 1989;63:3822-8

[8] Jacobson SG, Cideciyan AV, Roman AJ, et al. Improvement and decline in vision with gene therapy in childhood blindness. N Engl J Med 2015;372:1920-6

[9] Kotterman MA, Chalberg TW, Schaffer DV. Viral vectors for gene therapy: translational and clinical outlook. Annu Rev Biomed Eng 2015;17:63-89

[10] Nathwani AC, Reiss UM, Tuddenham EG, et al. Long-term safety and efficacy of factor IX gene therapy in hemophilia B. N Engl J Med 2014;371:1994-2004

[11] Mendell JR, Sahenk Z, Malik V, et al. A Phase 1/2a follistatin gene therapy trial for becker muscular dystrophy. Mol Ther 2015;23:192-201 • This article reports BMD proof-ofprinciple clinical trial.

[12] Srivastava A. In vivo tissue-tropism of adeno-associated viral vectors. Curr Opin Virol 2016;21:75-80

[13] Duan D, Sharma P, Yang J, et al. Circular intermediates of recombinant adeno-associated virus have defined structural characteristics responsible for long-term episomal persistence in muscle tissue. J Virol 1998;72:8568-77

[14] McCarty DM, Monahan PE, Samulski RJ. Self-complementary recombinant adenoassociated virus (scAAV) vectors promote efficient transduction independently of DNA synthesis. Gene Ther 2001;8:1248-54

[15] Hagedorn C, Schnödt M, Boehme P, et al. S/MAR element facilitates episomal long-term persistence of Adeno-associated viral (AAV) vector genomes in proliferating cells. Hum 
Gene Ther 2017;28:1169-79

[16] Flanigan KM. The Muscular Dystrophies. Semin Neurol 2012;32:255-63

[17] Koenig M, Hoffman EP, Bertelsons CJ, et al. Complete cloning of the duchenne muscular dystrophy (DMD) cDNA and preliminary genomic organization of the DMD gene in normal and affected individuals. Cell 1987;50:509-17

[18] Hoffman EP, Brown RH, Kunkel LM. Dystrophin: the protein product of the Duchenne muscular dystrophy locus. Cell 1987;51:919-28

[19] England SB, Nicholson LV, Johnson MA, et al. Very mild muscular dystrophy associated with the deletion of $46 \%$ of dystrophin. Nature 1990;343:180-2 • First observation indicates micro-dystrophin gene replacement for Duchenne muscular dystrophy.

[20] Clemens PR, Krause TL, Chan S, et al. Recombinant truncated dystrophin minigenes: construction, expression, and adenoviral delivery. Hum Gene Ther 1995;6:1477-85

[21] Gregorevic P, Allen JM, Minami E, et al. Systemic delivery of rAAV6-microdystrophin preserves muscle function and extends lifespan in a murine model of severe muscular dystrophy. Nat Med 2006;12:787-9

[22] Wang B, Li J, Xiao X. Adeno-associated virus vector carrying human minidystrophin genes effectively ameliorates muscular dystrophy in mdx mouse model. PNAS 2000;97:13714-9

[23] Watchko J, O’Day T, Wang B, et al. Adeno-associated virus vector-mediated minidystrophin gene therapy improves dystrophic muscle contractile gunction in mdx mice. Hum Gene Ther 2002;13:1451-60

[24] Yue Y, Pan X, Hakim CH, et al. Safe and bodywide muscle transduction in young adult Duchenne muscular dystrophy dogs with adeno-associated virus. Hum Mol Genet 2015; 24 : 5880-90

[25] Mendell JR, Campbell K, Rodino-Klapac L, et al. Dystrophin immunity in duchenne's muscular dystrophy. N Engl J Med 2010;363:1429-37 •• First gene therapy trial for DMD and highlights the presence of immune responses to the transgene or vector.

[26] Bowles DE, McPhee SW, Li C, et al. Phase 1 gene therapy for duchenne muscular dystrophy using a translational optimized AAV vector. Mol Ther 2012;20:443-55

[27] Yue Y, Ghosh A, Long C, et al. A single intravenous injection of adeno-associated virus serotype-9 leads to whole body skeletal muscle transduction in dogs. Mol Ther 2008;16:1944-52

[28] Kornegay JN, Li J, Bogan JR, et al. Widespread muscle expression of an AAV9 human minidystrophin vector after intravenous injection in neonatal dystrophin-deficient dogs. Mol Ther 2010;18:1501-8

[29] Koo T, Okada T, Athanasopoulos T, et al. Long-term functional adeno-associated virusmicrodystrophin expression in the dystrophic CXMDj dog. J Gene Med 2011;13:497-506

[30] Le Guiner C, Servais L, Montus M, et al. Long-term microdystrophin gene therapy is effective in a canine model of Duchenne muscular dystrophy. Nat Commun 2017: published online 25 July 2017, doi: 10.1038/ncomms 16105

[31] Wang Z, Kuhr CS, Allen JM, et al. Sustained AAV-mediated dystrophin expression in a canine model of duchenne muscular dystrophy with a brief course of immunosuppression. 
Mol Ther 2007;15:1160-6

[32] Rodino-Klapac LR, Montgomery CL, Bremer WG, et al. Persistent expression of FLAGtagged micro dystrophin in nonhuman primates following intramuscular and vascular delivery. Mol Ther 2010;18:109-17

[33] Xu R, Camboni M, Martin PT. Postnatal overexpression of the CT GalNAc transferase inhibits muscular dystrophy in mdx mice without altering muscle growth or neuromuscular development: evidence for a utrophin-independent mechanism. Neuromuscul Disord 2007; $17: 209-20$

[34] Tsuchida K. Myostatin inhibition by a follistatin-derived peptide ameliorates the pathophysiology of muscular dystrophy model mice. Acta Myol 2008;27:14-8

[35] Odom GL, Gregorevic P, Allen JM, et al. Microutrophin delivery through rAAV6 increases lifespan and improves muscle function in dystrophic dystrophin/utrophin-deficient mice. Mol Ther 2008;16:1539-45

[36] Pisani C, Strimpakos G, Gabanella F, et al. Utrophin up-regulation by artificial transcription factors induces muscle rescue and impacts the neuromuscular junction in mdx mice. Biochim Biophys Acta 2018: published online April 2018, doi: 10.1016/j.bbadis.2018.01.030

[37] Chicoine LG, Rodino-Klapac LR, Shao G, et al. Vascular Delivery of rAAVrh74.MCK.GALGT2 to the gastrocnemius muscle of the rhesus macaque stimulates the expression of dystrophin and laminin $\alpha 2$ surrogates. Mol Ther 2014;22:713-24

[38] McPherron AC, Lawler AM, Lee S-J. Regulation of skeletal muscle mass in mice by a new TGF-beta superfamily member. Nature 1997;387:83-90

[39] Kota J, Handy CR, Haidet AM, et al. Follistatin gene delivery enhances muscle growth and strength in nonhuman primates. Sci Transl Med 2009;1:6-15

[40] Haidet AM, Rizo L, Handy C, et al. Long-term enhancement of skeletal muscle mass and strength by single gene administration of myostatin inhibitors. PNAS 2008;105:4318-22

[41] Amthor H, Macharia R, Navarrete R, et al. Lack of myostatin results in excessive growth but impaired force generation. PNAS 2007;104:1835-40

[42] Mariot V, Joubert R, Hourdé C, et al. Downregulation of myostatin pathway in neuromuscular diseases may explain challenges of anti-myostatin therapeutic approaches. Nat Commun 2017: published online 30 Noveber 2017, doi:10.1038/s41467-017-01486-4

[43] Dumonceaux J, Marie S, Beley C, et al. Combination if myostatin pathway interference and dystrophin rescue enhances tetanic and specific force in dystrophic mdx mice. Mol Ther 2010;18:881-7

[44] Nelson CE, Hakim CH, Ousterout DG, et al. In vivo genome editing improves muscle function in a mouse model of Duchenne muscular dystrophy. Science 2016;351:403-7

[45] Tabebordbar M, Zhu K, Cheng JKW, et al. In vivo gene editing in dystrophic mouse muscle and muscle stem cells. Science 2016;351:407-11 - This article shows the potential of transduction of muscle stem cells with CRISPR/Cas tool.

[46] Long C, Amoasii L, Mireault AA, et al. Postnatal genome editing partially restores dystrophin expression in a mouse model of muscular dystrophy. Science 2016;351:400-3

[47] El Refaey M, Xu L, Gao Y, et al. In vivo genome editing restores dystrophin expression and 
cardiac function in dystrophic mice. Circ Res 2017:121:926-9

[48] Pini V, Morgan JE, Muntoni F, O'Neill HC. Genome editing and muscle stem cells as a therapeutic tool for muscular dystrophies. Curr Stem Cell Rep 2017;3:137-48

[49] Bengtsson NE, Hall JK, Odom GL, et al. Muscle-specific CRISPR/Cas9 dystrophin gene editing ameliorates pathophysiology in a mouse model for Duchenne muscular dystrophy. Nat Commun 2017: published online 23 June 2017, doi: 10.1038/ncomms16007

[50] Wang JZ, Wu P, Shi ZM, et al. The AAV-mediated and RNA-guided CRISPR/Cas9 system for gene therapy of DMD and BMD. Brain Dev 2017;39:547-56

[51] Cirak S, Arechavala-Gomeza V, Guglieri M, et al. Exon skipping and dystrophin restoration in patients with Duchenne muscular dystrophy after systemic phosphorodiamidate morpholino oligomer treatment: An open-label, phase 2, dose-escalation study. Lancet 2011;378:595-605

[52] Cirak S, Feng L, Anthony K, et al. Restoration of the dystrophin-associated glycoprotein complex after exon skipping therapy in duchenne muscular dystrophy. Mol Ther 2012;20:462-7

[53] Goemans NM, Tulinius M, van den Hauwe M, et al. Long-term efficacy, safety, and pharmacokinetics of drisapersen in duchenne muscular dystrophy: results from an open-label extension study. PLoS One 2016: published online 2 September 2016, doi: 10.1371/journal.pone.0161955

[54] Stefanovic B, Hackl W, Lührmann R, Schümperli D. Assembly, nuclear import and function of U7 snRNPs studied by microinjection of synthetic U7 RNA into Xenopus oocytes. Nucleic Acids Res 1995;23:3141-51

[55] Goyenvalle A, Vulin A, Fougerousse F, et al. Rescue of Dystrophic Muscle Through U7 snRNA-Mediated Exon Skipping. Science 2004;306:1796-9

[56] Goyenvalle A, Babbs A, Wright J, et al. Rescue of severely affected dystrophin/utrophindeficient mice through scAAV-U7snRNA-mediated exon skipping. Hum Mol Genet 2012;21:2559-71

[57] Bish LT, Sleeper MM, Forbes SC, et al. Long-term restoration of cardiac dystrophin expression in golden retriever muscular dystrophy following rAAV6-mediated exon skipping. Mol Ther 2012;20:580-9

[58] Vulin A, Barthélémy I, Goyenvalle A, et al. Muscle function recovery in golden retriever muscular dystrophy after AAV1-U7 exon skipping. Mol Ther 2012;20:2120-33

[59] Lefebvre S, Bürglen L, Reboullet S, et al. Identification and characterization of a spinal muscular atrophy-determining gene. Cell 1995;80:155-65

[60] Foust KD, Nurre E, Montgomery CL, et al. Intravascular AAV9 preferentially targets neonatal neurons and adult astrocytes. Nat Biotechnol 2009;27:59-65

[61] Foust KD, Wang X, McGovern VL, et al. Rescue of the spinal muscular atrophy phenotype in a mouse model by early postnatal delivery of SMN. Nat Biotechnol 2010;28:271-4

[62] Valori CF, Ning K, Wyles M, et al. Systemic delivery of scAAV9 expressing SMN prolongs survival in a model of spinal muscular atrophy. Sci Transl Med 2010;2:35-42

[63] Benkhelifa-Ziyyat S, Besse A, Roda M, et al. Intramuscular scAAV9-SMN injection 
mediates widespread gene delivery to the spinal cord and decreases disease severity in SMA mice. Mol Ther 2013;21:282-90

[64] Dominguez E, Marais T, Chatauret N, et al. Intravenous scAAV9 delivery of a codonoptimized SMN1 sequence rescues SMA mice. Hum Mol Gent 2011;20:681-93

[65] Passini MA, Bu J, Richards AM, et al. Translational fidelity of intrathecal delivery of selfcomplementary AAV9-survival motor neuron 1 for spinal muscular atrophy. Hum Gene Ther 2014;25:619-30

[66] Duque SI, Arnold WD, Odermatt P, et al. A large animal model of spinal muscular atrophy and correction of phenotype. Ann Neurol 2015;77:399-414

[67] Finkel RS, McDermott MP, Kaufmann P, et al. Observational study of spinal muscular atrophy type I and implications for clinical trials. Neurology 2014;83:810-7

[68] Mendell JR, Al-Zaidy S, Shell R, et al. Single-dose gene-replacement therapy for spinal muscular atrophy. N Engl J Med 2017;377:1713-22 • This article reports the promising outcomes achieved from the phase 1 clinical trial in SMA type 1 patients.

[69] Hinderer C, Katz N, Buza EL, et al. Severe toxicity in nonhuman primates and piglets following high-dose intravenous administration of an adeno-associated virus vector expressing human SMN. Hum Gene Ther 2018;29:285-98 • This article reports the toxicity of systemic high-dose AAV administration in large animals.

[70] Hordeaux J, Wang Q, Katz N, et al. The Neurotropic Properties of AAV-PHP.B Are Limited to C57BL/6J Mice. Mol Ther 2018;26:664-8

[71] Flotte TR, Büning H. Severe Toxicity in Nonhuman Primates and Piglets with Systemic High-Dose Administration of Adeno-Associated Virus Serotype 9-Like Vectors: Putting Patients First. Hum Gene Ther 2018;29:283-4

[72] Lorson CL, Androphy EJ. An exonic enhancer is required for inclusion of an essential exon in the SMA-determining gene SMN. Hum Mol Genet 2000;9:259-65

[73] Nirmal K, Singh NN, Androphy EJ, Singh RN. Splicing of a critical exon of human survival motor neuron is regulated by a unique silencer element located in the last intron. Mol Cell Biol 2006;26:1333-46

[74] Finkel RS, Mercuri E, Darras BT, et al. Nusinersen versus sham control in infantile-onset spinal muscular atrophy. N Engl J Med 2017;377:1723-32 • This article summarizes the promising results obtained with Nusinersen that have led to its approval by the FDA as first drug for the treatment of SMA disease.

[75] Gorman L, Mercatante DR, Kole R. Restoration of correct splicing of thalassemic $\beta$-globin pre-mRNA by modified U1 snRNAs. J Biol Chem 2000;275:35914-9

[76] Dal Mas A, Rogalska ME, Bussani E, Pagani F. Improvement of SMN2 pre-mRNA processing mediated by exon-specific U1 small nuclear RNA. Am J Hum Genet 2015;96:93103

[77] Marquis J, Meyer K, Angehrn L, et al. Spinal muscular atrophy: SMN2 pre-mRNA splicing corrected by a U7 snRNA derivative carrying a splicing enhancer sequence. Mol Ther 2007; $15: 1479-86$

[78] Odermatt P, Trüb J, Furrer L, et al. Somatic therapy of a mouse SMA model with a U7 snRNA gene correcting SMN2 splicing. Mol Ther 2016;24:1797-1805 
[79] Mayer K, Ferraiuolo L, Schmelzer L, et al. Improving single injection CSF delivery of AAV9-mediated gene therapy for SMA: a dose-response study in mice and nonhuman primates. Mol Ther 2015;23:477-87

[80] Victor M, Hayes R, Adams RD. Oculopharyngeal muscular dystrophy. A familial disease of late life characterized by dysphagia and progressive ptosis of the eyelids. N Engl J Med 1962;267:1267-72

[81] Brais B, Bouchard JP, Xie YG, et al. Short GCG expansions in the PABP2 gene cause oculopharyngeal muscular dystrophy. Nat Genet 1998;18:164-7

[82] Tomé FM Fardeau M. Nuclear inclusions in oculopharyngeal dystrophy. Acta Neuropathol 1980;49:85-7

[83] Malerba A, Klein P, Bachtarzi H, et al. PABPN1 gene therapy for oculopharyngeal muscular dystrophy. Nat Commun 2017: 8:14848. • This article reports the dual-AAV gene therapy approach for OPMD.

[84] Strings V, Malerba A, Harbaran S, et al. "Silence and Replace": Development of a single AAV vector system for the treatment of oculopharyngeal muscular dystrophy (OPMD). Hum Gene Ther 2017;12:A1-A125

[85] McEntagart M, Parsons G, Buj-Bello A, et al. Genotype-phenotype correlations in X-linked myotubular myopathy. Neuromuscul Disord 2002;12:939-46

[86] Laporte J, Hu LJ, Kretz C, et al. A gene mutated in X-linked myotubular myopathy defines a new putative tyrosine phosphatase family conserved in yeast. Nat Genet 1996;13:175-82

[87] Cui X, De Vivo I, Slany R, et al. Association of SET domain and myotubularin-related proteins modulates growth control. Nat Genet 1998;18:331-7

[88] Taylor GS, Maehama T, Dixon JE. Myotubularin, a protein tyrosine phosphatase mutated in myotubular myopathy, dephosphorylates the lipid second messenger, phosphatidylinositol 3phosphate. PNAS 2000;97:8910-5

[89] Gurgel-Giannetti J, Zanoteli E, de Castro Concentino EL, et al. Necklace fibers as histopathological marker in a patient with severe form of X-linked myotubular myopathy. Neuromuscul Disord 2012;22:541-5

[90] Buj-Bello A, Fougerousse F, Schwab Y, et al. AAV-mediated intramuscular delivery of myotubularin corrects the myotubular myopathy phenotype in targeted murine muscle and suggests a function in plasma membrane homeostasis. Hum Mol Genet 2008;17:2132-43

[91] Childers MK, Joubert R, Poulard K, et al. Gene therapy prolongs survival and restores function in murine and canine models of myotubular myopathy. Sci Transl Med 2014: published online 22 January 2014, doi: 10.1126/scitranslmed.3007523

[92] Mack DL, Poulard K, Goddard MA, et al. Systemic AAV8-mediated gene therapy drives whole-body correction of myotubular myopathy in dogs. Mol Ther 2017;25:839-54

[93] Hollinger K, Chamberlain JS. Viral vector-mediated gene therapies. Curr Opin Neurol 2015;28:522-7

[94] Sintusek P, Catapano F, Angkathunkayul N, et al. Histopathological defects in intestine in severe spinal muscular atrophy mice are improved by systemic antisense oligonucleotide treatment. PLoS One 2016: published online 10 May 2016, doi:

10.1371/journal.pone.0155032 
[95] Somers E, Lees RD, Hoban K, et al. Vascular defects and spinal cord hypoxia in spinal muscular atrophy. Ann Neurol 2016;79:217-30

[96] Hamilton G, Gilingwater TH. Spinal muscular atrophy: going beyond the motor neuron. Trends Mol Med 2013;19:40-50

[97] Zhou H, Meng J, Marrosu E, et al. Repeated low doses of morpholino antisense oligomer: an intermediate mouse model of spinal muscular atrophy to explore the window of therapeutic response. Hum Mol Genet 2015;24:6265-77

[98] Goncalves MA, van Nierop GP, Tijssen MR, et al. Transfer of the full-length dystrophincoding sequence into muscle cells by a dual high-capacity hybrid viral vector with sitespecific integration ability. J Virol 2005;79:3146-62

[99] Lostal W, Kodippili K, Yue Y, Duan D. Full-length dystrophin reconstitution with adenoassociated viral vectors. Hum Gene Ther 2014;25:552-62

[100] Koo T, Popplewell L, Athanasopoulos T, Dickson G. Triple trans-splicing adeno-associated virus vectors capable of transferring the coding sequence for full-length dystrophin protein into dystrophic mice. Hum Gene Ther 2014;25:98-108 


\section{Figures legends}

Figure 1: AAV transduction. (1) After the injection, ssAAV or a scAAV vectors are imported inside the cell by internalization in clathrin coated endocytic vesicles (2-3). The vectors are then released from the vesicles into the cytoplasm (4) translocating to the nucleus where transgenes are released (5). Once inside the nucleus, the DNA from ssAAV and scAAV vectors undergo different processes. Within ssAAV vector, a single DNA strand is transported and requires the synthesis of a second strand inside the nucleus to form dsDNA(6), while in the scAAV vector this step is bypassed since both DNA stands are packaged and transported. The dsDNA released from either vector remains in transduced cells in episome state, and only a very small percentage $(0.1-1 \%)$ of vector genome is integrated into the host chromosome (7), followed by the transgene expression in transduced cells (8).

Figure 2: AAV- mediated gene therapy in DMD. Duchenne muscular dystrophy is caused by mutations in dystrophin gene leading to the disruption of the open reading frame followed by a nonfunctional protein production (shown in light red). Different AAV-mediated gene therapy approaches have been designed, some of those act through dystrophin protein while others do not target directly the dystrophin protein. (A) Two different approaches of AAV-mediated gene therapy acting through dystrophin gene: gene replacement and exon skipping. Gene replacement approach consists on the introduction of micro-dystrophin gene packaged into AAV vectors. Exon skipping allows restoring dystrophin expression with a truncated but functional dystrophin protein. Two strategies have been designed. One strategy uses engineered U7 snRNA to carry specific exonskipping AONs delivered by AAV vectors and the second one relies on CRISPR/Cas9 technology, where CRISPR/Cas9 complexes are delivered by dual- or single-AAV vectors system. (B) Surrogate gene therapy strategies for DMD treatment. These include the overexpression of proteins such as GALG2 enzyme responsible for glycosylation of $\alpha$-dystroglycan in skeletal muscle and the 
muscle growth stimulating protein follistatin.

Figure 3: AAV-mediated gene therapy in SMA. Spinal muscular atrophy is caused by the loss-offunction mutations in SMN1 gene, while SMN2 is intact in all patients and only $10 \%$ of SMN2 trascripts is full-length and can be translated into a functional SMN protein (shown in yellow). To date two AAV-gene therapy approaches have been validated: SMN1 gene replacement and AAVmediated antisense oligonucleotide approach. In the SMN1-AAV gene replacement approach, the introduction of SMN1 allows the production of full-length SMN protein. The second strategy targets SMN2 gene by using engineered U7 or U1 snRNA to carry specific AONs delivered by AAV vectors. AONs are designed to augment exon 7 splicing in SMN2 gene and increase the fulllength SMN transcripts and functional SMN protein.

Figure 4: AAV-mediated gene therapy in OPMD. A17 mouse model of OPMD expresses a mutated PABPN1 with 17 alanine residues, in addition to the endogenous PABPN1. This mouse model displays several clinical symptoms of OPMD, such as the presence of nuclear aggregates and muscle atrophy and weakness. The dual-AAV approach, which consists of knocking down the endogenous PABPN1 by shRNA (scAAV-shRNA3X) and replacing the protein with a wild-type PABPN1 (scAAV-optPABPN1), has been developed by the findings of the single approach experiments. In the approach of PABPN1 knockdown only, the INIs are removed but no amelioration in muscle degeneration is observed. Similarly in the approach of wild-type PABPN1 replacement only, no improvement in muscle pathology is observed in A17 mouse model. The combination of these two strategies using two AAV vectors leads to a significant rescue of the phenotype. The optimization of this strategy has been conducted by Benitec Biopharma using a single vector system (BB-301) to accomplish both approaches as a potential treatment for OPMD patients. The first clinical trial is due to start in 2018 . 